\title{
Modelling Gait Processes as a Combination of Sensory-motor and Cognitive Controls in an Attempt to Describe Accidents on the Level in Occupational Situations
}

\author{
Alexandrine SICRE1, Sylvie LECLERCQ ${ }^{2 *}$, Clarisse GAUDEZ², Gabriel M. GAUTHIER ${ }^{1}$, \\ Jean-Louis VERCHER ${ }^{1}$ and Christophe BOURDIN ${ }^{1}$
}

\author{
${ }^{1}$ UMR $6152<<$ Mouvement et Perception>> Faculté des Sciences du Sport 163, avenue de Luminy-CP 910 \\ 13288 Marseille cedex 09, France \\ ${ }^{2}$ Département Homme au travail, Laboratoire de Biomécanique et d'Ergonomie INRS — Avenue de Bourgogne- \\ BP 2754501 Vandoeuvre cedex, France
}

Received June 28, 2007 and accepted October 12, 2007

\begin{abstract}
In occupational situations, accidents referred to as accidents on the level (AoLs) occur most of the time when locomotion control fails. This control is determined by the interactions between the operator and the environment, the task and the used tools. Hence, AoLs prevention requires developing ways to optimise these interactions. More fundamentally, AoLs prevention requires understanding locomotion control in situations where this control is at sake, that is in situations involving one or more AoLs factors. The purpose of this article is to propose a comprehensive model for the control of locomotion in occupational environments. This model featuring the operator, the task and the working space should be an appropriate tool to understand AoLs in the scope of their prevention. Firstly, we describe what occupational AoLs are. In a second part, we present a review of the theoretical and experimental knowledge related to the locomotion system through the various means developed by the Central Nervous System to cope with perturbations of the environment and/or particular constraints from the task. Finally, we propose a simplified systemic model presenting the various levels of control (sensory-motor to cognitive levels) describing locomotion in occupational situations, and we suggest experiments likely to produce the appropriate data to construct the final comprehensive model.
\end{abstract}

Key words: Accident on the level, Prevention, Gait adaptation, Strategic control, Adaptive control, Cognitive level, Sensory-motor level

\section{Introduction}

The purpose of this article is to design a comprehensive model for the control of locomotion in occupational situations. The available literature concerning the accidents by fall relates, by a majority, to accidents involving elderly people (exceeding $70 \mathrm{yr}$ ). Explanatory factors are found in the intrinsic characteristics of the individual such as the evolution with age of the threshold of visual sensitivity ${ }^{1}$, or the postural anxiety (fear of falling)

*To whom correspondence should be addressed. consecutive to a previous accident ${ }^{2}$. Leclercq $^{3)}$ showed that the factors describing accidents occurring in occupational situations are to be sought not exclusively in a greater individual susceptibility to fall (for example, people in occupational situations are likely to be different age group) but more likely in the disturbances related to the interactions between the individual and the components of his occupational situation, i.e. the environment, the task and the used tools. French statistics applying to occupational accidents also accredit this assumption. Indeed, the employees below $30 \mathrm{yr}$ of age present the higher risk to be injured due to accidents on the level 
(AoLs) and exposure to risk varies according to concerned activity sectors ${ }^{4}$. The variability of exposure according to the branch of activity was also reported by Buck and Coleman $^{5)}$ and Kemmlert and Lundholm ${ }^{6}$.

\section{The Accidents On-level in Occupational Situations}

In France, the employee national health insurance fund (Caisse Nationale d'Assurance Maladie des travailleurs Salariés, CNAMTS) insures 18 million employees. Results derived from employees' declared occupational accidents point out that AoLs are ranked second among all occupational accidents with loss of working days. These accidents represented, in 2005, 24\% of all declared occupational accidents with at least $24 \mathrm{~h}$ off-work (e.g. 165,734 accidents with days lost), $22 \%$ of occupational accidents leading to permanent disablement (e.g. 11,442 accidents leading to permanent disablement) and were the cause of $25 \%$ of days lost due to temporary disablement $(8,216,240$ days lost due to temporary disablement).

Analyses of AoLs which have occurred in several French companies reveal that these accidents, for the majority, are caused by an unexpected disturbance of balance which does not systematically involve a fall and which occurs on an even or slightly irregular surface such as a sidewalk, pavement, or inclined plane ${ }^{7,8)}$. These accidents or part of them are designed in the international literature as "slips and trips"9), or "falls"10), or "slips"11).

Among the 83,255 occupational accidents registered in Sweden during the first half of the year of 1979, 9,725 were slips (i.e. $12 \%$ of all occupational accidents). 59\% of these slips occurred during normal walking ${ }^{12}$. However, it is in only $14 \%$ of 459 cases of AoLs particularly serious which have occurred in France and recorded in the data base EPICEA ${ }^{13)}$ that the activity of the victim was coded as "walking"14). In this specific database, the activity can be described as "walking", "inspecting", "evacuating". One cannot thus exclude that the victim was walking when the coded activity was "inspecting" for example. That is confirmed by systematic analyses of AoLs carried out in some companies which show a higher frequency of AoLs during operators' displacement, this displacement sometimes occurring while the operator carries out another activity $\left.{ }^{7}, 15,16\right)$. As all the occupational accidents, AoLs are the consequence of a combination of accident factors which concern all the components of the occupational situation. Fig. 1, named "causal tree", represents two examples of the logical combination of events leading to AoL. Identified factors are, in these examples, the physical state of the ground (slipperiness, smoothness, regularity...), the time constraint applied on the operator, and the attentional load required by the task.

These factors considered in isolation do not systematically result in an accident ${ }^{17)}$. Still, one may wonder why these combined factors could affect the regular control of locomotion. The comprehension of the control of balance during locomotion, carried out or not at the same time as another physical and/or cognitive activity, thus appears essential within the framework of the study of these accidents.

As already mentioned earlier, AoLs in occupational situations occur most of the time when locomotion control fails. However, this control is determined by the interactions between the operator and the environment, the task and the used tools. For example, carrying out a task under time constraints or in an unknown environment or when visual attention is already focused on something in the environment can prevent the detection of an obstacle when walking. The conception and the state of the physical environment or the presence of obstruction are also elements which may contribute to AoLs. Hence, AoLs prevention requires developing ways to optimise the interactions between the operator, the environment, the task and the tools, and therefore understanding locomotion control in situation where this control is at sake, that is in situation involving one or more AoLs factors.

Over the last $10 \mathrm{yr}$, ergonomics studies addressing specifically the question of AoLs have revealed aspects of human behaviour which must be taken into account when modelling locomotion in a complex, changing environment. For example, the study by Gao et Abeysekera ${ }^{18)}$ has shown that, in presence of snow or ice on the ground, the occurrence of a fall, and postural balance recovery after major instability ${ }^{19-21)}$, depends on previous experience. Thus according to these authors "training in gait balance may help minimize slip and fall risks on ice for inexperienced outdoor workers and pedestrians". Other studies describe the incidence of biomechanical parameters on AoLs when carrying a load ${ }^{22,23)}$, at various speeds $^{22}$, for various ground inclinations and slipperiness ${ }^{23,24)}$, while taking a turn on more or less slippery grounds ${ }^{25}$, when the operator is or not aware of the slipperiness of the ground ${ }^{26)}$ or when the operator has or not experienced the slippery ground. The most often studied interaction between human and environment is the shoe/slippery floor interaction when walking. The authors pointed out the increased risk of slipping as the friction requirement when performing a task (walking, load carrying, pushing/pulling) exceed the available friction of the shoe/floor interface.

These biomechanical studies have mainly led to recommendations related to the use of anti-slip footwear and the installation of an anti-slip floor covering as well as to the development of slipperiness measurement device. 

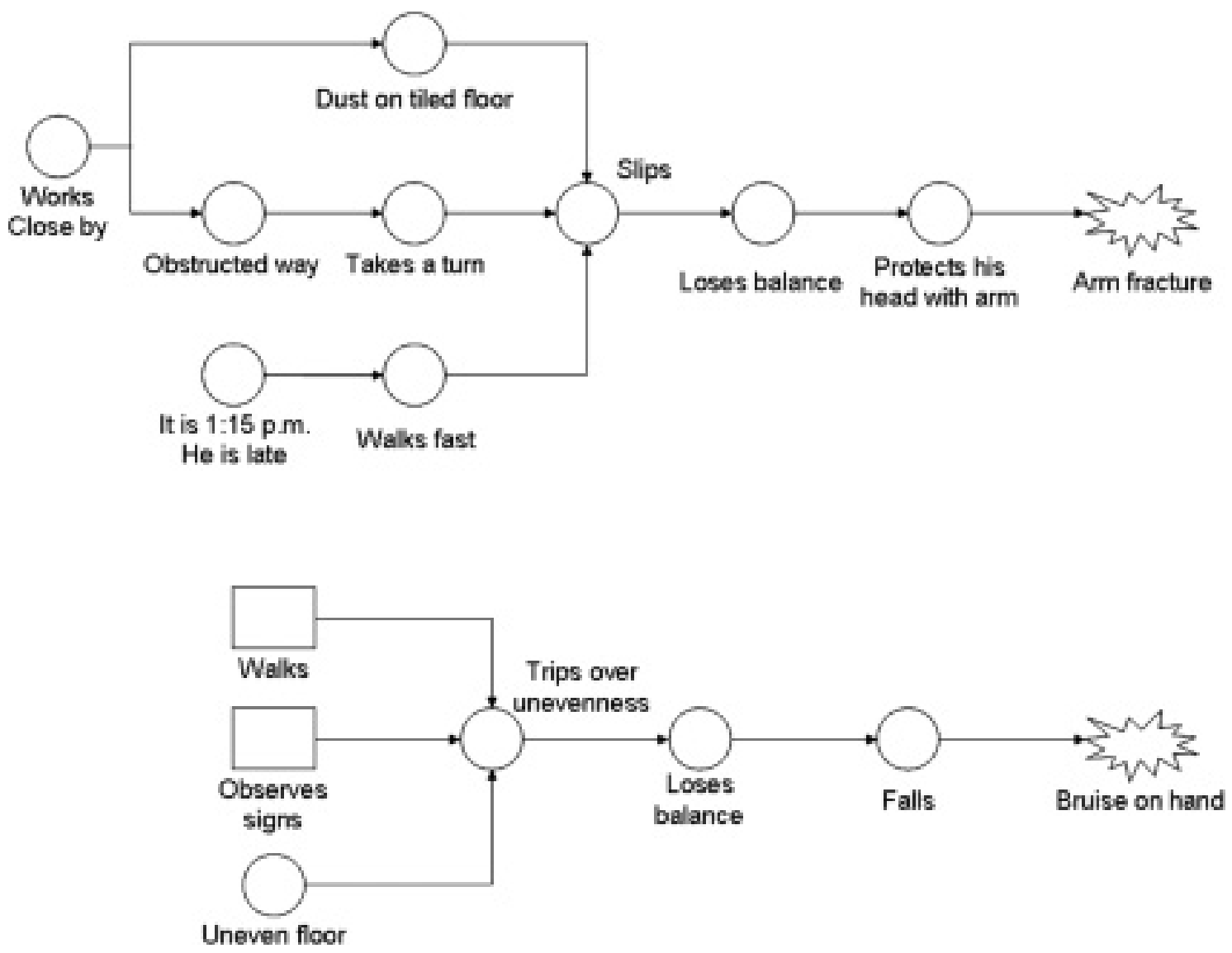

Fig. 1. Examples of $<<$ causal tree $>>$ (logical sequence of events, 17) leading to AoLs in occupational situations. Squares represent regular events and Circles represent variations from usual.

However, studies devoted to prevent the slipping are insufficient to respond to the prevention requirements of all occupational AoLs. Other studies aiming at understanding the recovery processes after balance disturbances cannot describe why the balance control failed. Consequently, understanding the recovery processes and describing the biomechanical constraints imposed by a slippery surface represent a particular way to analyze the AoLs but remains incomplete and insufficient to explain them as a whole.

Let's imagine an operator walking on a corridor with a slippery surface localized at the end of this corridor. Consider now that the operator has all the needed sensory information to see and anticipate for the perturbation induced by this hazardous zone. Nonetheless, it may be that, sometimes, the anticipatory adjustment of the locomotor pattern will not be appropriately done, potentially leading to AoL. The question to be solved to explain the occurrence of AoLs in such situations requires to describe the processes activated by the subjects to cope with the environmental constraints and the task constraints to adjust the locomotor patterns. The basic idea of such a point of view is that the occurrence of AoLs will reveal that some of these processes fail. In this review, we will stress the control processes activated before the balance disturbance rather than after the perturbation. In other words, we emphasize all control processes activated before the time of occurrence of perturbation (that is before reaching the perturbing area) rather than on the recovery processes activated after the perturbation.

This ambitious project necessitates to closely understanding the processes underlying locomotion. We propose that one way to understand how AoLs occur is to assemble in a single model both the basic knowledge regarding locomotion function and the various factors affecting locomotion in occupational situations. These factors will be connected with the environment, the task carried out and the operator. The model should then describe locomotion instabilities occurring in conditions where the operator in normal physical state, having access to a full set of information and resources commonly used to control locomotion, does not anticipate appropriately a perturbation. The consequent unexpected postural instability eventually ends as a fall or any kind of musculoskeletal lesions generally described by the terms AoL.

The systemic model will take into account not only low 


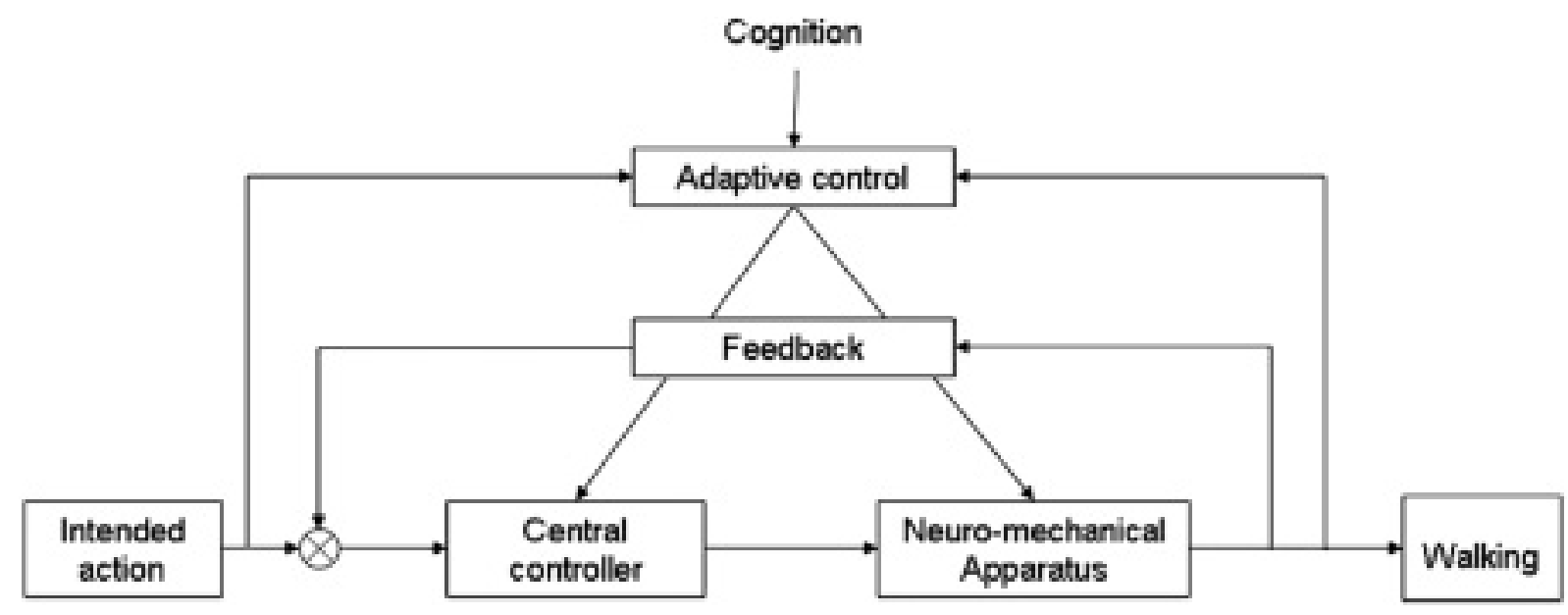

Fig. 2. Basic model of the locomotion system featuring sensorimotor and cognitive level controls. The main feedback (low level sensorimotor control, called online control) combines information from visual, vestibular, proprioceptive and tactile origins.

level sensory-motor processes but also elaborated higher level cognitive processes (Fig. 2).

The review attempts to finally propose a model of the organization of the motor behaviour which could be expanded through locomotor task, to explain the processes leading to adjusted behaviour in an anticipatory way.

\section{Appropriate Description of AoL Requires Full Knowledge of Locomotion Function}

Although the biomechanical analysis of walking is quite complex, the fact that spinalized animals can produce a stepping motion, that looks like normal gait, implies that the generation of a stepping pattern may in some ways be simple ${ }^{27)}$. Nevertheless, these same animals do require complementary stabilization, suggesting that control of balance during gait is a more complex issue than is generation of the stepping pattern. Indeed, Bauby and $\mathrm{KuO}^{28)}$ proposed a model of locomotion suggesting that active control from higher centres is necessary at least for lateral stabilization of gait.

From a motor control point of view, the occurrence of AoLs during locomotion phases reveals that some central control processes were inefficient or failed. We question these processes arising before the occurrence of the balance disturbances. In that way, we are concerned in the way the brain mobilizes resources to predict and anticipate, then avoid, the effects of a potential perturbation. What are the control processes involved in this behaviour?

Walking is a complicated motor act requiring the coordination of trunk and limb muscles, crossing many joints. How the nervous system manages to accomplish this complex task has intrigued scientists for years. In a normal and highly predictable environment, locomotor synergies involving several muscles may take place at lower spinal level with neural circuitry tuned by local loops of assistance or self-organizing processes generated in coordinative networks. Neural networks in the spinal cord, referred to as "Central Pattern Generators" (CPGs), are capable of producing rhythmic movements, such as swimming, walking, and hopping, even when isolated from the brain and sensory inputs ${ }^{29}$. It has become clear, however, that in the intact nervous system, CPGs do not operate in a vacuum but depend on the interplay of information between the brain and spinal cord, with the final motor output shaped by sensory feedback from peripheral receptors and reconfigured by neuromodulators ${ }^{29}$. Indeed, basic locomotion resulting from a CPG command can be modulated by a descending command from the motor cortex, as revealed by the observed increased discharge in motor cortical units in cats modifying their gait to meet environmental demands ${ }^{30-34)}$. Microstimulation of the motor cortex in cats ${ }^{35,36)}$ and transcranial magnetic stimulation in humans ${ }^{37}$, both applied at different phases of the step cycle, have been shown to produce a phasedependent effect on locomotion: rather than being purely imposed on the CPG command, the descending command from the motor cortex thus appears to functionally interact with the CPG to adapt basic locomotion. In that sense, study of locomotion could not be restricted to the analysis of the CPGs mechanisms.

Seen differently, locomotion is a complex mechanism calling for both sensory motor and cognitive processes. Lajoie et al. ${ }^{38)}$ have shown that upright standing and walking tasks require the integration of several sources of sensory information. It is now well-known that locomotion is mainly regulated online by visual, proprioceptive, vestibular and tactile information. When a perturbation arises suddenly, online control mechanisms are activated 
to maintain balance. Moreover, when the perturbation is repeated (imagine an operator going through the same slippery surface each day), additional controls can step in, mainly adaptive control at sensorimotor level, and strategic control at cognitive level (such as to carry out the task another way $)^{39,40)}$. This multi-level approach of locomotion production and control could be summarized by suggesting that stability during locomotion is maintained through reactive, predictive, and anticipatory strategies ${ }^{41}$ ) which we shall describe in similar terms more appropriate to a systemic approach, namely online, adaptation and strategic controls, although these later terms are not equivalent one to one to the earlier terms.

\section{Online control of locomotion}

Extensive research on locomotor control over the past decades has elucidated a crucial role of afferent input in shaping the motor output during walking. Information are provided by visual, vestibular, tactile and proprioceptive systems.

Vision plays a critical role in the maintenance of locomotor control ${ }^{42)}$. For example, the motor command producing the locomotor pattern is directly linked to optical information in such a way that the system can continuously evaluate the current situation and update the command ${ }^{43)}$. Prokop et al. ${ }^{44)}$ studied the influence of vision on human locomotion. Subjects had to walk on a moving treadmill while optic flow was provided. This optic flow (see ${ }^{45)}$ for description of optic flow) was displayed at many velocities, going from adjusted to the walking velocity to displayed backward, three times faster than walking velocity. The results showed that forward optic flow lead to an increase of walking velocity whereas backward optic flow lead to a decrease of walking velocity, a linear relationship linking optic flow and walking velocity. Optic flow was also demonstrated to induce a modification in stride length/stride frequency ratio, describing a linear relationship between walking velocity and optic flow characteristics. More accurately, results showed optic flow induce more modulation of stride length than stride frequency, indicating that "space-related parameter is influenced by visually perceived motion information, whereas the temporal parameter remains stable".

Afferent signals from muscle receptors are also important factors for timing the transition between stance and swing, and for regulating the magnitude of muscle activity during the stance phase ${ }^{46-48)}$. Other factors are involved Perry et al. ${ }^{49)}$ and Perry ${ }^{50)}$ have demonstrated that plantar mechanoreceptors were also involved in the control of locomotion while this one was perturbed in an unpredictable multi-directional way. Subjects with sustained hypothermic anaesthesia of the foot soles, had to face forward, backward and lateral translations of the platform. The results showed that afferents coming from plantar cutaneous mechanoreceptors have a specific direction and phase dependant role.

\section{Adaptive control}

Adaptive control refers to a brain process which allows sensory-motor systems to maintain or recover a fairly high performance level in spite of changes affecting the usual relationship between the sensory input and the motor output. It implies that neural networks in the CNS are plastically modifiable. Applied to our subject of concern, it describes the observation that during locomotion, if a recurrent alteration occurs, the central nervous system will have to generate or remodel the motor output in relation to the alteration. An influential concept is that the nervous system uses internal models of the dynamics of the body parts involved in a movement to define the motor command and to predict the sensory signals that will income as a consequence of the action ${ }^{51)}$ (Fig. 3).

Then, from the desired state, the system will internally represent the coming transformation, considering the dynamics of the limbs and environment. It is thought that the nervous system is capable of predicting the forces that will be experienced during upcoming movement to produce the appropriate motor commands ${ }^{52}$. This prediction is based on an internal representation, or model, of the dynamic properties of the limb related to the environment. Thus when an alteration such as the application of an external force arises, the dynamical properties of the limb are modified, resulting in errors in movement kinematics. Through experience, i.e. when the alteration is maintained, the internal model is updated so that the motor commands are modified to account for alterations in movement condition ${ }^{52,53)}$. On return to the original movement condition, the modified motor commands will no longer be valid, again resulting in movement errors called after-effects ${ }^{52)}$. The presence of after-effects after removal of the alteration can be accounted for by the notion that the nervous system uses internal models to control and adapt movements ${ }^{54)}$.

Two internal models are specified. The first one is the inverse model, which defines the motor command considering the desired state. The second one is the forward model, besides called predictor, which can predict the consequences of a coming action (in terms of sensory) from a copy of the motor command (efference copy). Then, from sensory feedback, the predicted state is compared with the real state. If any differences, the adaptive controller, which gets an error signal, will work on inverse and forward models in purpose to update them considering the produced error. The interactions between the adaptive controller and the forward and inverse models 


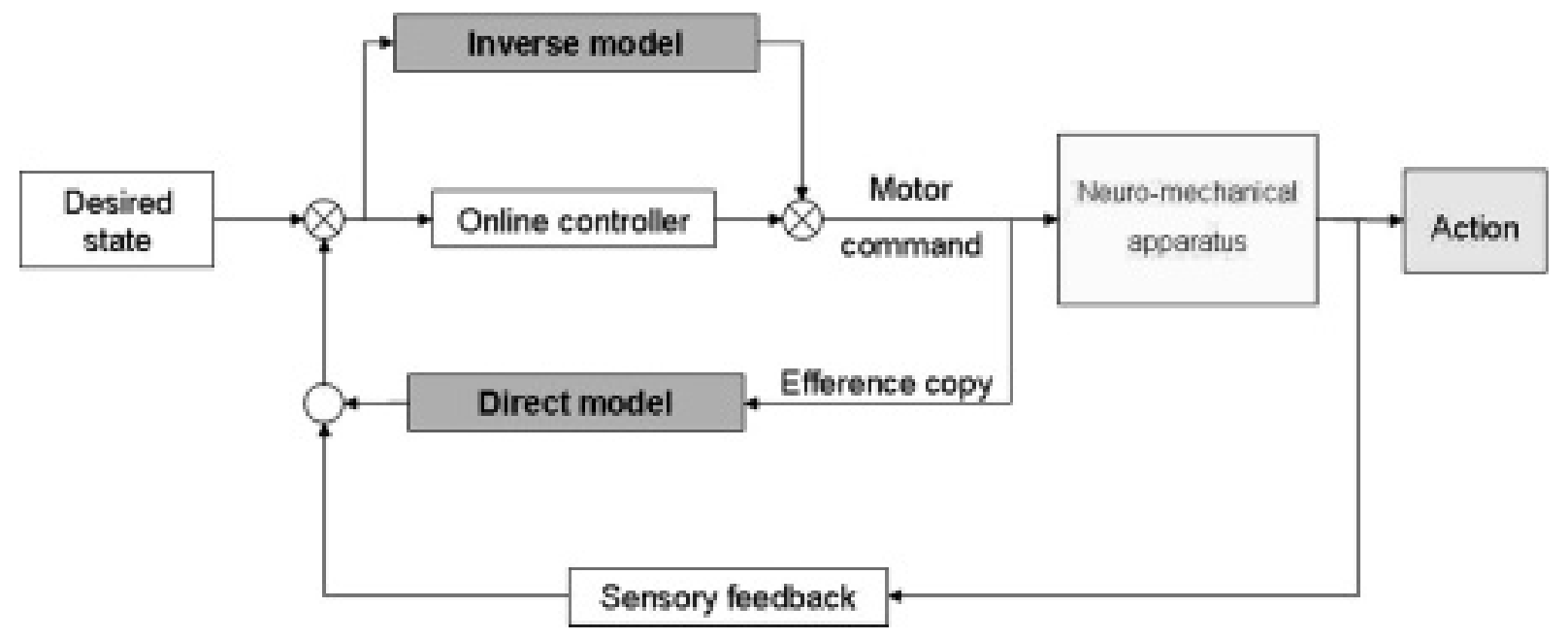

Fig. 3. Schematic representation of the inverse model and the direct model in the sensory-motor loop.

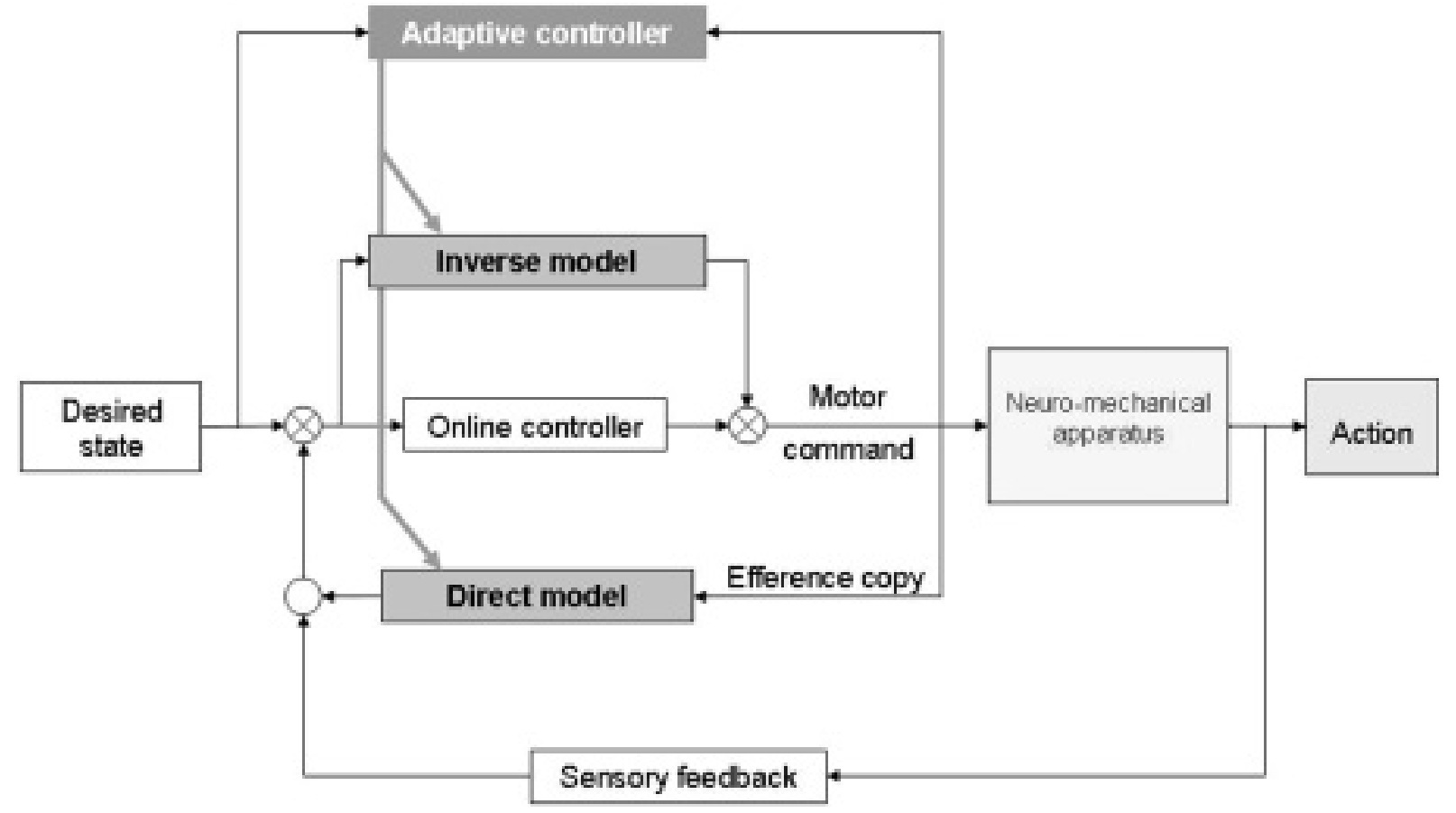

Fig. 4. Simplified model featuring the online controller and the adaptive controller.

are illustrated in Fig. 4.

Experimental evidences for the updating of the internal models for sensory-motor adaptation initially come from studies mainly interested in the production of pointing movements with the hand ${ }^{55-57)}$. Still, there is growing evidence that an internal model is also used in the control of gait. In fact, when subjects undergo a repeated alteration, the locomotor pattern progressively adapt until recovering a normal gait. Moreover, after-effects following the removal of the alteration are also described ${ }^{58-60)}$. A common example of adaptive control in the daily life is the "broken escalator phenomenon", namely the sensation that when walking onto an escalator which is stationary one experiences an odd sensation of imbalance, despite full awareness that the escalator is not going to move. That "false" (because unreal) sensation of sway can be considered as an after-effect of gait adaptation and would be, according to Reynolds et al. ${ }^{61)}$, elicited by an "inappropriate expression of a learned motor behaviour". Infant subjects ${ }^{59,60)}$ and adult subjects ${ }^{58)}$ adapt to the constant presence of a disturbance to swing phase movements and show after-effects on removal of the disturbance. There is also evidence for long-lasting modifications in interlimb coordination after 
a period of walking on a rotating disk ${ }^{62,63)}$ or a split-belt treadmill ${ }^{64)}$. Lam et al. ${ }^{65)}$ have applied a velocity-dependant resistance on one leg and observed that during the first steps, locomotion was perturbed, but returned pretty easily to a normal pattern. Then, when perturbation was removed, after-effects were observed.

The presence of after-effects after a period of training under new conditions implies the formation or recalibration of the motor output for a given task. Those aftereffects, which are in general disappearing ${ }^{1}$ with a shorter time course than the adaptation time course, are characteristics of adaptive control, that is to say updating of internal models involved in the task. Updating of internal models can be induced by different types of alteration as reported above. Reports mention velocity-dependant resistance applied to hip and knee ${ }^{65)}$, walking on a moving platform ${ }^{66)}$, walking onto a moving sled ${ }^{61)}$, reduction or increase in body weight ${ }^{64)}$. Gordon et al. ${ }^{62)}$ have conducted an experiment with human adults walking $2 \mathrm{~h}$ on a horizontally rotating disc inducing abnormal locomotion since the external leg must move faster than the inner leg. After that period of perturbation, the subjects with the vision occluded were asked to walk straight ahead. They showed after-effects, as evidenced by curvilinear trajectories as compared to data collected before walking on the disk. Previous works have also shown that blindfolded adults are able to walk straight ahead with accuracy $^{67)}$. In Jensen et al.'s study ${ }^{64)}$, curved trajectories were maintained during half an hour, nevertheless decreasing step by step.

It is worth noting that the term "adaptation" employed in the literature can refers to two different notions. The first and the most frequently used one refers to the change of the pattern to cope with a single current alteration. In this case, the locomotor system does not undergo internal changes whatsoever. The online control alone or the online control assisted by the strategic control (which might solve the locomotion problem another way) handles the situation. The second use of the term "adaptation", more rarely utilized, refers to the updating of internal models, in other words, to the adaptive control. Then, several studies referring to "adaptation" have nothing to do with internal models, particularly biomechanics and psychological locomotion studies.

\section{Strategic control}

Natural walking is the result of online (automatic) sensory-motor control, usually sufficient to maintain balance. Although locomotion is basically automatic, it can be intentionally modulated ${ }^{68)}$ as in daily life situations when somebody's walking calls for cognitive intervention.

\footnotetext{
${ }^{1}$ Traditionally, reduction of the after-effect is called extinction.
}

Then, locomotion becomes the result of the integration of strategic, adaptive and online controls, the contribution of each level (sensory-motor and cognitive levels) varying according to the situation. Here, we shall define cognitive control as a high level control contributing to planning, producing, and perceiving actions with the subject's will and awareness. Psychologists ${ }^{69)}$ tend to define cognitive actions as actions that can be described verbally by the actor. Strategic control is part of cognitive control. Since the human body is constituted of more degrees of freedom than needed, multiples solutions can apply to the resolution of the same problem or to generate the same action. Hence, out of a repertoire of possibilities, the brain chooses the most appropriate possibility (strategy) to solve a given problem occurring at a given time, relying on various information from the body, the environment, previous experience, etc. Merleau-Ponty ${ }^{70)}$ already considered locomotion under its cognitive aspect: "since in the neuronal activity our least conscious reactions can never be isolated, and since these reactions always seem to be guided by the internal as well as the external situation and are able, up till a certain point, to adapt to any particularity of these situations, we can no longer maintain the classical clear-cut distinction between reflex activity and instinctive or intelligent activity: we cannot oppose blind automatism to intentional activity, between which the relation would stay, by the way, unclear".

Let's consider the situation where a single alteration occurs (as opposed to a recurrent alteration treated earlier which may activate the adaptive control). If the alteration is predictable and predicted, the cognitive system might call for strategic control. The strategic control, based on previous experience and knowledge, will infer the properties of the coming alteration and choose a way to deal with it to provide the best locomotor pattern and trajectory to maintain balance. Richards $e t$ al. ${ }^{71)}$ define strategic control as "the process of introducing on-line adjustments, or interventions, to mitigate the consequences of erroneous motor outputs inappropriate for some altered sensory environment". Strategic control occurs early in the adaptation process, once the subject becomes aware of a coming sensory discordance and decides at some conscious level how to correct for it ${ }^{72-74)}$. For instance, after the onset of a visual discordance (for instance by decorrelating the optic flow from the direction of locomotion), increased variability is observed in gait coordination patterns reflecting the use of strategic control mechanisms by the CNS in an attempt to resolve the conflict between visual, vestibular, and proprioceptive cues and motor output ${ }^{75-77)}$. 
Relationships between adaptive control and strategic control

It is interesting to note that according to the experiments, subjects can be warned or not of the occurring and the later removing of the alteration. When there is advance warning of removing of the alteration subjects still generate after-effects ${ }^{61,62)}$. We can then easily deduce that such after-effects are the result of the updating of internal models (adaptive control). Indeed, it cannot be simply the result of strategic control. Then and as proposed by Richards et al. ${ }^{71)}$, strategic control and adaptive control are two distinct processes working in parallel to plastically modify locomotion (in opposite to online control). Their results suggest that adaptation involves a continuous interplay between strategic and adaptive modifications of motor control. Usually, these controls are thought to be activated at different levels of the CNS, that is the sensory-motor level and the cognitive level. Once again, adaptation of the gait parameters seems to involve both levels of control. Strategic control belongs to the cognitive level (conscious) while adaptive control belongs to the sensory-motor level (unconscious). As mentioned earlier, adaptive control is likely to be mediated by the cognitive level.

It is also interesting to note that strategic control and adaptive control differ according to the level of consciousness involved in the task. An obvious example of this fact, though not involving locomotion, is the experience conducted by Ingram et al. ${ }^{78)}$, in which a perturbation impacted the visuo-motor system. One part of the task consisted in pointing a visual target with one hand. Visual feedback was not direct, but provided by a cursor display. At the beginning of the experience (pre-exposure period), the cursor display was faithful to reality. For the exposure period, there were two conditions in which the cursor display did not reflect reality anymore; in the first one, the gain of the cursor changed progressively, twisting the visuo-motor relation in a manner that subjects did not realise it. In the second condition, the gain of the cursor display was abruptly changed, in a manner that subjects definitely perceived it. The final gain for the two conditions was the same. At the post-exposure period (gain value is one), the results showed that for the first condition, there was after-effects that progressively extinguished, characteristic of internal models updating, whereas for the second condition, there was no aftereffects, indicating that subjects performed the task thanks to strategic control only. Then it appears that internal models updating is prevented, or at least slowed down, by the involvement of consciousness. Still, other experiments may demonstrate that consciousness may contribute to faster adaptation, keeping open the question as to the influence of consciousness in adaptation control.
In complement, some studies have shown that in a dual task situation, there is a prioritisation of the postural control at the expense of the cognitive task. In Faulkner et $a l .{ }^{16)}$ study on elderly, during concurrent reaction time task and walking, results pointed out an increase of reaction time whereas walking time was preserved. As shown previously in Brown et al. ${ }^{79)}$ study, prioritisation can be modified in situation of postural threat. Indeed, they quantified the relationship between postural control and the second (cognitive) task performance, and have shown that subjects prioritised more the postural control over the second task when threatened, particularly in elderly compared to young. In the same way, Gage et al. ${ }^{80)}$ have shown that reaction times increase when walking in the condition of greatest postural threat. Generally, most of the studies have shown that the allocation of cognitive resources to postural control or locomotion varies with the age of the subjects - increasing for elderly ${ }^{81,82)}$ and the nature of the cognitive tasks ${ }^{83)}$ added to the postural or locomotion task.

\section{Modelling Gait Control Processes}

Based on the assumption that AoLs in occupational situations occur when locomotion control fails (that is when the gait pattern is no more adjusted to the constraints), the present review is aimed to detail the theoretical framework useful to describe locomotion control. The basic statement concerning AoLs in working environments suggests that these accidents can occur when several accident factors combine (see Fig. 1 for the description of AoLs). In addition, these accidents can occur even if the operator was confronted many times with the same situation, and even if the operator has access to the full set of information allowing anticipating for the perturbation. How can the occurrence of AoLs be explained by theoretical knowledge on the processes underlying the production and the adjustment of locomotion?

This striking and motivating question represents a major challenge for researchers in motor control. To give a satisfactory answer to this crucial question posed by people in charge of prevention, we have reviewed, in the previous section, numerous experimental studies devoted to the analysis and the understanding of these processes of control. Rather than describing the recovery phase, we are particularly interested in explaining how the operators in occupational situations may anticipate for some perturbations to preserve their physical integrity and to avoid AoLs. In this way, we have described two different levels of control, the sensory-motor and the cognitive level, involving different types of control including the online, the adaptive and the strategic controls. We now propose a model (Fig. 5) of these processes of control, based on 


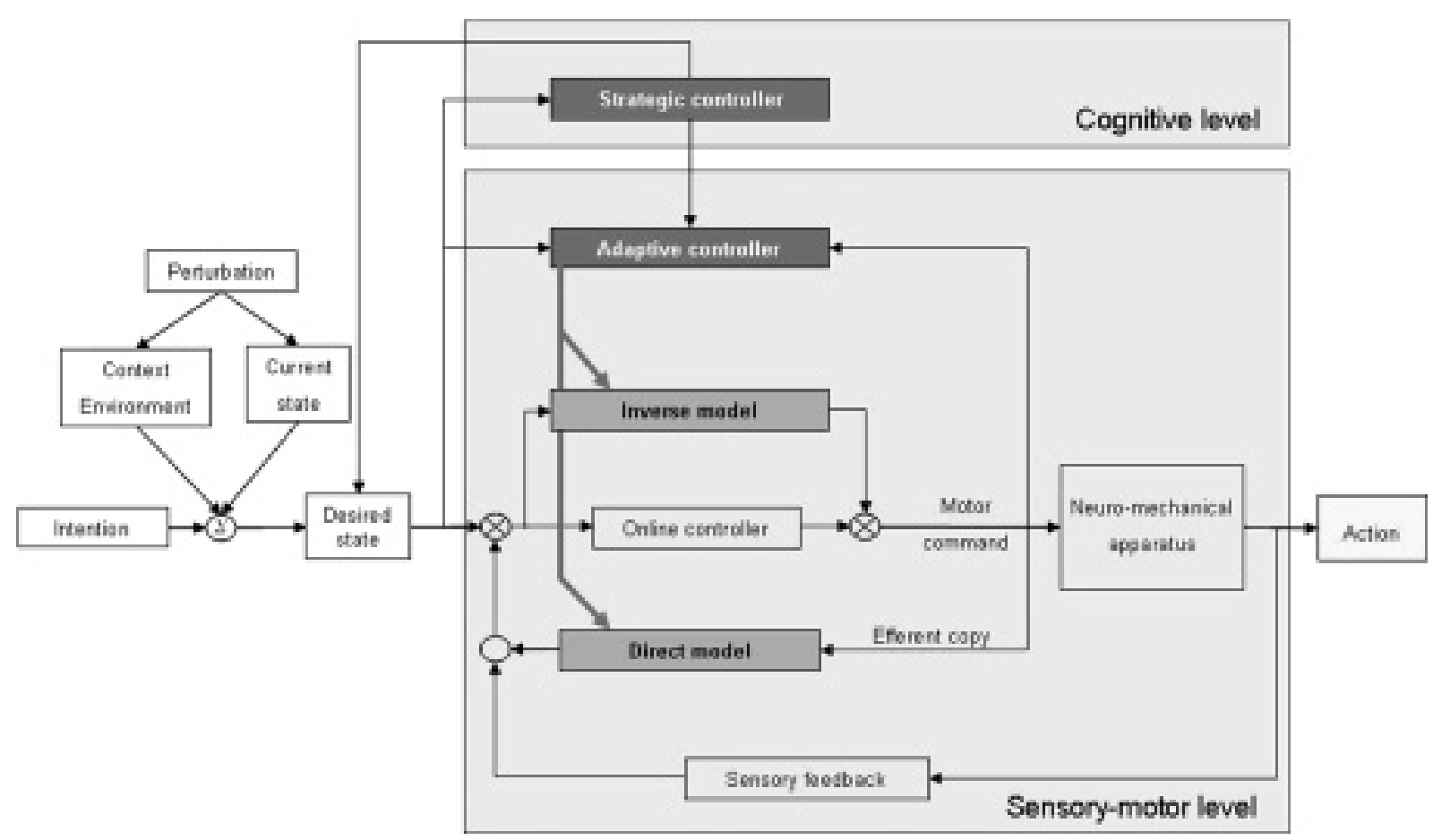

Fig. 5. Simplified systemic model representing the three basic controllers (online, adaptive and strategic) and the two levels of control (sensory-motor and cognitive).

the basic one presented in Fig. 2. The model features online control, adaptive control and strategic control as we assume three controls to develop, the online control to handle normal situations, adaptive control to take into account the internal changes resulting from the alterations affecting locomotion, and strategic control to integrate physical environment characteristics and operator state.

The main purpose of this model is to provide a synthesised description of the processes activated during the motor adaptation of the gait pattern, by summarising data obtained in previous experiment. We propose to model sensory-motor and cognitive processes with particular emphasis on their interaction. The model is not designed to represent the locomotion system in its biomechanical aspects nor its neural anatomical details. It will not then be usable to describe the mechanisms by which a subject recovers balance or falls after a balance disturbance, nor the inappropriate response of the locomotion function due to unexpected events originated within the overall locomotion system. The model will rather be designed to represent the basic control mechanisms likely to be activated while an operator walks in a structured environment in which predictable or unpredictable events may occur either singularly or repeatedly. The model will feature two major interacting control levels (sensory-motor and cognitive).

How does this model describe an every day activity? The walker's online controller, as mentioned previously, allows regulating the locomotion directly from the sensory feedback provided by the system. For example, while crossing the street, the online controller will regulate the length of the steps, starting a few steps before the sidewalk, to allow the walker to systematically step over the sidewalk with the same leg (usually the dominant leg). The online controller is always activated, even when adaptive and strategic controllers step in.

The adaptive controller progressively modifies, through the plasticity of the CNS, the internal models (direct and inverse) when a mismatch is detected between desired and real state. For example, when somebody wears ski shoes which are heavier and more rigid than regular shoes, the locomotion is first altered then progressively evolves towards close-to-normal locomotion (in spite of not using ankle rotations). The adaptive controller has updated the leg internal model which now takes into account both the new dynamics of the legs and the physical impossibility to rely on ankle rotation. After removing the shoes, the walker will experience a disturbed walking for a few steps showing that indeed, the internal model had been modified. The strategic control belongs to cognitive level. It allows choosing a way of realising an action. For example, when someone is walking on a straight and flat way with only one obstacle on it, the subject can choose to step over the obstacle or to circle it. 


\section{Conclusions}

Studies of processes activated in the production and adjustment of gait parameters provide a promising approach for learning about sensory-motor and cognitive aspects of locomotion control. It also offers a new way for people in charge of prevention to get knowledge and understanding to cope with the AoLs in occupational situations. This motor control approach has to be complementary of the biomechanical studies describing the recovery processes or aimed at producing data to develop slip resistance (of shoes and/or floors) measurement device or slip resistance safety threshold to cope with AoLs in specific occupational situations. Even if this kind of approach may be promising and mostly innovative, it firstly necessitates to synthesize the data collected in the past. For this, we have proposed a synthetic model of control of locomotion, based on an abundant literature focused principally on gait analysis. The next step of this exciting work will concern the experimental validation of the model. In the future, we will be particularly interested in the way the strategic and the adaptive controls may cooperate for producing balanced locomotion. We assume that detailed analysis of locomotor outputs in response to alterations of the environment, the task or the body allows a close look at sensory motor and cognitive processes. A particular and crucial development of this model will concern the way the operators deal with discrete perturbation of locomotion (a small slippery surface along a long corridor for example). We have already chosen to tackle the overall problem using a sliding paradigm to collect new data on cognitive control of locomotion (the less documented part of the field) in hazardous environment, likely to complete modelling of locomotion. On the long range, we hope that this model will be used to improve the occupational situations and to reduce significantly the occurrence of the AoLs.

\section{Acknowledgement}

Alexandrine Sicre was supported by grant NO 5063233 from Institut National de Recherche et de Sécurité (INRS).

\section{References}

1) Van Dieën JH, Pijnappels M, Bobbert MF (2005) Agerelated intrinsic limitations in preventing a trip and regaining balance after a trip. Safety Sci 43, 437-53.

2) Brown L, Doan J, McKenzie N, Cooper S (2006) Anxiety-mediated gait adaptations reduce errors of obstacle negotiation among younger and older adults: Implications for fall risk. Gait Posture 24, 418-23.
3) Leclercq S (2002) Prevention of falls on the level in occupational situations: a major issue, a risk to be managed. Int J Occup Saf Ergon 8, 377-85.

4) Gaudez C, Leclercq S (2007) Accident de plain-pied. Données statistiques et analyses menées en entreprises. Document pour le Médecin du Travail (in French).

5) Buck PC, Coleman VP (1985) Slipping, tripping and falling accidents at work: a national picture. Ergonomics 28, 949-58.

6) Kemmlert K, Lundholm L (2001) Slips, trips and falls in different work groups-with reference to age and from a preventive perspective. Appl Ergon 32, 149-53.

7) Leclercq S, Thouy S (2004) Systemic analysis of socalled "accidents on the level" in a multi trade company. Ergonomics 47, 1282-300.

8) Leclercq S, Thouy S, Rossignol E (2007) Progress in understanding processes underlying occupational accidents on the level based on case studies. Ergonomics 1, 59-79.

9) Health and Safety Executive Preventing slips and trips in the workplace. http://www.hse.gov.uk/slips/index.htm. Accessed September 13, 2007.

10) Nagata $H$ (1991) Analysis of fatal falls on the same level or on stairs/steps. Safety Sci 14, 213-22.

11) Gronqvist R, Roine J (1993) Serious occupationnal accidents caused by slipping. In: Advances in Industrial Ergonomics and Safety V, Nielsen R and Jorgensen K (Eds.), 515-9, Taylor and Francis, London.

12) Strandberg L, Lanshammar $H$ (1981) The dynamics of slipping accidents. J Occup Accidents 3, 153-62.

13) Ho MT, Bastide JC, Francois C (1986) Mise au point d'un système destiné à l'exploitation de comptes rendus d'analyse d'accidents du travail. Trav Humain 49, 137-46 (in French).

14) Leclercq S, Tissot C (2004) Serious falls on the level in occupational situations. In: Contemporary Ergonomics, Mc Cabe (Eds.), 83-7, CRC Press, London.

15) Bentley TA, Haslam RA (1998) Slip, trip and fall accidents occurring during the delivery of mail. Ergonomics 41, 1859-72.

16) Faulkner KA, Redfern MS, Rosano C, Landsittel DP, Studenski SA, Cauley JA, Zmuda JM, Simonsick EM, Kritchevsky SB, Newman AB, Study HA (2006) Reciprocal influence of concurrent walking and cognitive testing on performance in older adults. Gait Posture 24, 182-9.

17) Monteau M (1997) Analysis and reporting accident investigation. In: Encyclopaedia of Occupational Health and Safety, 1, 57.22-5, B.I.T., Geneve.

18) Gao C, Abeysekera J (2004) Slips and falls on ice and snow in relation to experience in winter climate and winter sport. Safety Sci 42, 537-45

19) Brady RA, Pavol MJ, Owings TM, Grabiner MD (2000) Foot displacement but not velocity predicts the outcome of a slip induced in young subjects while walking. $\mathrm{J}$ Biomech 33, 803-8.

20) Cham R, Redfern MS (2001) Lower extremity correc- 
tive reactions to slip events. J Biomech 34, 1439-45.

21) You J, Chou Y, Lin C, Su F (2001) Effect of slip on movement of body center of mass relative to base of support. Clin Biomech 16, 167-73.

22) Hsiang SM, Chang C (2002) The effect of gait speed and load carrying on the reliability of ground reaction forces. Safety Sci 40, 639-57.

23) Cham R, Redfern MS (2004) Gait adaptations during load carrying on level and inclined surfaces. Occup Ergon 4, 11-26.

24) Cham R, Redfern MS (2002) Heel contact dynamics during slip events on level and inclined surfaces. Safety Sci 40, 559-76.

25) Chiou SS, Bhattacharya A, Lai CF, Succop PA (2003) Effects of environmental and job-task factors on workers' gait characteristics on slippery surfaces. Occupational Ergonomics 3, 209-23.

26) Chambers AJ, Margerum S, Redfern MS, Cham R (2003) Kinematics of the foot during slips. Occup Ergon 3, 225-34.

27) Grillner S, Wallen $P$ (1985) Central pattern generators for locomotion, with special reference to vertebrates. Annu Rev Neurosci 8, 233-61.

28) Bauby CE, Kuo AD (2000) Active control of lateral balance in human walking. J Biomech 33, 1433-40.

29) MacKay-Lyons M (2002) Central pattern generation of locomotion: a review of the evidence. Phys Ther 82, 69-83.

30) Beloozerova IN, Sirota MG (1998) Cortically controlled gait adjustments in the cat. Ann N Y Acad Sci 860, 550-3.

31) Drew T (1988) Motor cortical cell discharge during voluntary gait modification. Brain Res 457, 181-7.

32) Drew T (1991) Visuomotor coordination in locomotion. Curr Opin Neurobiol 1, 652-7.

33) Drew T (1993) Motor cortical activity during voluntary gait modifications in the cat. I. Cells related to the forelimbs. J Neurophysiol 70, 179-99.

34) Amos A, Armstrong DM, Marple-Horvat DE (1990) Changes in the discharge patterns of motor cortical neurones associated with volitional changes in stepping in the cat. Neurosci Lett 109, 107-12.

35) Armstrong DM, Drew T (1985) Forelimb electromyographic responses to motor cortex stimulation during locomotion in the cat. J Physiol 367, 327-51.

36) Kalaska JF, Drew T (1993) Motor cortex and visuomotor behavior. Exerc Sport Sci Rev 21, 397-436.

37) Schubert M, Curt A, Jensen L, Dietz V (1997) Corticospinal input in human gait: modulation of magnetically evoked motor responses. Exp Brain Res 115, 234-46.

38) Lajoie Y, Teasdale N, Bard C, Fleury M (1993) Attentional demands for static and dynamic equilibrium. Exp Brain Res 97, 139-44.

39) Werner S, Bock O (2007) Effects of variable practice and declarative knowledge on sensorimotor adaptation to rotated visual feedback. Exp Brain Res 178, 554-9.
40) Newport R, Jackson SR (2006) Posterior parietal cortex and the dissociable components of prism adaptation. Neuropsychologia 44, 2757-65.

41) Patla AE (2003) Strategies for dynamic stability during adaptive human locomotion. IEEE Eng Med Biol Mag 22, 48-52.

42) Gibson J (1966) The senses considered as a perceptual system, MIT Press, Cambridge.

43) de Rugy A, Taga G, Montagne G, Buekers MJ, Laurent M (2002) Perception-action coupling model for human locomotor pointing. Biol Cybern 87,141-50.

44) Prokop T, Schubert M, Berger W (1997) Visual influence on human locomotion. Modulation to changes in optic flow. Exp Brain Res 114, 63-70.

45) Gibson JJ (1979) The Ecological Approach to visual Perception, Houghton-Mifflin, Boston.

46) Dietz V, Muller R, Colombo G (2002) Locomotor activity in spinal man: significance of afferent input from joint and load receptors. Brain 125, 2626-34.

47) Nielsen JB, Sinkjaer T (2002) Afferent feedback in the control of human gait. J Electromyogr Kinesiol 12, 213-7.

48) Pearson KG (2004) Generating the walking gait: role of sensory feedback. Prog Brain Res 143, 123-9.

49) Perry SD, McIlroy WE, Maki BE (2000) The role of plantar cutaneous mechanoreceptors in the control of compensatory stepping reactions evoked by unpredictable, multi-directional perturbation. Brain Res 877, 401-6.

50) Perry SD, Santos LC, Patla AE (2001) Contribution of vision and cutaneous sensation to the control of centre of mass (COM) during gait termination. Brain Res 913, 27-34.

51) Kawato M, Furukawa K, Suzuki R (1987) A hierarchical neural-network model for control and learning of voluntary movement. Biol Cybern 57, 169-85.

52) Shadmehr R, Mussa-Ivaldi F (1994) Adaptive representation of dynamics during learning of a motor task. J Neurosci Soc Neurosci 14, 3208-24.

53) Thoroughman KA, Shadmehr R (1999) Electromyographic correlates of learning an internal model of reaching movements. J Neurosci 19, 8573-88.

54) Kawato M (1999) Internal models for motor control and trajectory planning. Curr Opin Neurobiol 9, 718-27.

55) Bourdin C, Bringoux L, Gauthier GM, Vercher JL (2006) Vision of the hand prior to movement onset allows full motor adaptation to a multi-force environment. Brain Res Bull 71, 101-10.

56) Lackner JR, DiZio P (1992) Gravitoinertial force level affects the appreciation of limb position during muscle vibration. Brain Res 592, 175-80.

57) Shadmehr R (2004) Generalization as a behavioral window to the neural mechanisms of learning internal models. Hum Mov Sci 23, 543-68.

58) Emken JL, Reinkensmeyer DJ (2005) Robot-enhanced motor learning: accelerating internal model formation during locomotion by transient dynamic amplification. 
IEEE Trans Neural Syst Rehabil Eng 13, 33-9.

59) Lam T, Wolstenholme C, Yang JF (2003) How do infants adapt to loading of the limb during the swing phase of stepping? J Neurophysiol 89, 1920-8.

60) Pang MY, Lam T, Yang JF (2003) Infants adapt their stepping to repeated trip-inducing stimuli. J Neurophysiol 90, 2731-40.

61) Reynolds RF, Bronstein AM (2003) The broken escalator phenomenon. Aftereffect of walking onto a moving platform. Exp Brain Res 151, 301-8.

62) Gordon CR, Fletcher WA, Melvill Jones G, Block EW (1995) Adaptive plasticity in the control of locomotor trajectory. Exp Brain Res 102, 540-5.

63) Weber KD, Fletcher WA, Gordon CR, Melvill Jones G, Block EW (1998) Motor learning in the "podokinetic" system and its role in spatial orientation during locomotion. Exp Brain Res 120, 377-85.

64) Jensen L, Prokop T, Dietz V (1998) Adaptational effects during human split-belt walking: influence of afferent input. Exp Brain Res 118, 126-30.

65) Lam T, Anderschitz M, Dietz V (2006) Contribution of feedback and feedforward strategies to locomotor adaptations. J Neurophysiol 95, 766-73.

66) Bunday KL, Reynolds RF, Kaski D, Rao M, Salman S, Bronstein AM (2006) The effect of trial number on the emergence of the 'broken escalator' locomotor aftereffect. Exp Brain Res 174, 270-8.

67) Rieser JJ, Ashmead DH, Talor CR, Youngquist GA (1990) Visual perception and the guidance of locomotion without vision to previously seen targets. Perception 19, 675-89.

68) Rossignol S (1996) Visuomotor regulation of locomotion. Can J Physiol Pharmacol 74, 418-25.

69) Bridgeman B, Huemer V (1992) Conscious versus unconscious processes. The case of vision. Theory Psychol 2, 73-88.

70) Merleau-Ponty M (1942) La structure du comportement, Presses Universitaires de France, Paris (in French).

71) Richards JT, Mulavara AP, Bloomberg JJ (2007) The interplay between strategic and adaptive control mechanisms in plastic recalibration of locomotor function. Exp Brain Res 178, 326-38.
72) Redding GM, Wallace B (1996) Adaptive spatial alignment and strategic perceptual-motor control. J Exp Psychol Hum Percept Perform 22, 379-94.

73) McNay EC, Willingham DB (1998) Deficit in learning of a motor skill requiring strategy, but not of perceptuomotor recalibration, with aging. Learn Mem 4, 411-20.

74) Seidler RD, Noll DC, Thiers G (2004) Feedforward and feedback processes in motor control. Neuroimage 22, 1775-83.

75) Bardy BG, Warren WH Jr, Kay BA (1999) The role of central and peripheral vision in postural control during walking. Percept Psychophys 61, 1356-68.

76) Warren WH Jr, Kay BA, Zosh WD, Duchon AP, Sahuc $S$ (2001) Optic flow is used to control human walking. Nat Neurosci 4, 213-6.

77) Richards JT, Mulavara AP, Bloomberg JJ (2004) Postural stability during treadmill locomotion as a function of the visual polarity and rotation of a three-dimensional environment. Presence Teleoper Vir Real 13, 371-84.

78) Ingram HA, van Donkelaar $\mathrm{P}$, Cole $\mathrm{J}$, Vercher JL, Gauthier GM, Miall RC (2000) The role of proprioception and attention in a visuomotor adaptation task. Exp Brain Res 132, 14-26.

79) Brown LA, Sleik RJ, Polych MA, Gage WH (2002) Is the prioritization of postural control altered in conditions of postural threat in younger and older adults? J Gerontol A Biol Sci Med Sci 57, 785-92.

80) Gage W, Sleik R, Polych M, McKenzie N, Brown L (2003) The allocation of attention during locomotion is altered by anxiety. Exp Brain Res 150, 385-94.

81) Melzer I, Bejuya N, Kaplanski J (2001) Age-related changes of postural control: effect of cognitive tasks. Gerontology 47, 189-94.

82) Rankin JK, Woollacott MH, Shumway-Cook A, Brown LA (2000) Cognitive influence on postural stability: a neuromuscular analysis in young and older adults. $\mathbf{J}$ Gerontol A Biol Sci Med Sci 55, 112-9.

83) Maylor EA, Wing AM (1996) Age differences in postural stability are increased by additional cognitive demands. J Gerontol B: Psychol Sci Soc Sci 51, 143-54. 